\title{
In situ measurement and modelling of the growth and length scale of twins in $\alpha$-uranium
}

\author{
Nicolò Grilli $\odot,{ }^{1, *}$ Edmund Tarleton $\odot,{ }^{1,2, \dagger}$ Philip D. Edmondson, ${ }^{3}$ Maxim N. Gussev, ${ }^{4}$ and Alan C. F. Cocks $\oplus^{1}$ \\ ${ }^{1}$ Department of Engineering Science, University of Oxford, Parks Road, OX1 3PJ United Kingdom \\ ${ }^{2}$ Department of Materials, University of Oxford, Parks Road, OX1 3PH United Kingdom \\ ${ }^{3}$ Materials Science and Technology Division, Oak Ridge National Laboratory, Oak Ridge, Tennessee 37831, USA \\ ${ }^{4}$ Reactor and Nuclear Systems Division, Oak Ridge National Laboratory, Oak Ridge, Tennessee 37831, USA
}

(Received 13 December 2019; accepted 3 April 2020; published 27 April 2020)

\begin{abstract}
Twinning is an important deformation mechanism in crystalline materials. Twins nucleate, then grow, forming a discrete pattern of deformation bands with a certain thickness. The mechanism for twin growth is not completely understood. We present a simple phase field model which captures the physics of twin growth and reproduces the details observed during in situ electron backscatter diffraction experiments on $\alpha$-uranium. The interaction between residual dislocations at twin interfaces and mobile dislocations in untwinned regions increases the stress needed for twin growth. This is described by a nonlocal term in the proposed constitutive equations: the nucleation stress of a twin increases proportionally to the twin phase field in a neighborhood. Competition between slip and twinning favors the nucleation of a new twin rather than the growth of a preexisting twin. The phase field model is coupled with a crystal plasticity finite element solver that includes the plastic deformation due to twinning. Simulations are able to reproduce the number of twins and their thicknesses as a function of the strain observed during in situ electron backscatter diffraction experiments. The stress-strain curve is also reproduced. This comparison allows the values of the nucleation stress and the interaction strength between residual and mobile dislocations to be found. It also gives an estimation of the density of residual dislocations that is consistent with the observed twin thickness. This model can be applied to understand microstructural effects in materials using twinning as a strengthening mechanism.
\end{abstract}

DOI: 10.1103/PhysRevMaterials.4.043605

\section{INTRODUCTION}

Strain-induced twinning in crystalline materials is a deformation mechanism in which atoms form a mirror image of the original crystal when a certain level of stress is applied [1,2]. Twinning occurs in a wide variety of materials [3], particularly, when few slip systems are active and in low stacking fault energy metals [4].

Twinning is an important mechanism in materials engineering as it allows the microstructure to be modified. Twin interfaces are obstacles hindering the motion of dislocations and provide a strengthening mechanism in a similar manner to grain refinement $[5,6]$. Techniques to form nanotwinned grains have recently been developed, which lead to lightweight alloys that exhibit high strength and ductility $[7,8]$. However, the large homogeneous plastic shear induced by twinning leads to internal stresses which can initiate microcracks [9]. Therefore, computational material models which accurately capture the physics of twinning are essential.

Nucleation mechanisms for twins have been proposed for fcc and bcc metals, based on dislocation loops expanding on three neighboring atomic planes. Such a configuration of dislocation loops can be created by a jog dislocation (pole mechanism) [10] or by the climb motion of interacting partial dislocations (fault pair model) [11]. Molecular

\footnotetext{
*nicolo.grilli@eng.ox.ac.uk

†edmund.tarleton@eng.ox.ac.uk
}

dynamics (MD) simulations suggest that, after the nucleation stage, the motion of the dislocation loops, forming a socalled twinning dislocation, leads to twin propagation across a grain [12]. However, MD simulations require the use of very high strain rates, so caution is required when modeling strain rate-dependent problems, such as twinning.

After nucleation, twins grow up to a certain thickness that is dependent on the material and loading conditions. Some metals, such as $\mathrm{Mg}$, show a single large twin inside a grain after deformation, with a thickness of $10 \mu \mathrm{m}$ or more [13], whereas other materials, such as the metal of interest here, orthorhombic $\alpha$-uranium [14], form many micron-sized twins.

The mechanism that determines the twin thickness and spacing is still a debated issue. MD simulations have shown that the interaction of the twin interface with mobile dislocations or conjugate twins leads to the formation of new twin layers or to the translation of the twin interface [12]. These phenomena, called twin growth and migration, respectively, can increase the thickness of the twin. However, twin embryos growing layer by layer can become unstable after reaching a critical number of layers [15], making it necessary for multiple smaller embryos to merge before growth can take place [2]. Partial dislocations that form new twin layers can also be nucleated at a twin interface if the stress is sufficiently high [16]. Another aspect that needs to be clarified is the competition between deformation twinning and dislocation slip.

Discrete twins have been observed after deformation using transmission electron microscopy [17], and residual strains 
have been measured [18]. However, due to the high speed at which twins nucleate and propagate [19], the direct observation of these mechanisms and the determination of the stress necessary to induce them remains challenging.

This raises a number of important questions. Which mechanism is necessary to explain twin growth? What is the stress necessary to induce it? How does the presence of a twin affect the nucleation of a neighboring twin? What are the factors that determine twin thickness and spacing?

To answer these questions, we propose a continuum model for twin nucleation and growth that is validated using in situ electron backscatter diffraction (EBSD) experiments on $\alpha$ uranium. This paper introduces a nonlocal interaction term in the constitutive equations to describe the effects of preexisting twins and dislocations on the local twin nucleation stress. We compare, the dynamic formation of new twins, twin patterning, and the stress-strain response, using crystal plasticity simulations and in situ EBSD. These experiments provide the crystal orientation prior to the mechanical loading [20], and the crystal reorientation allows individual twins to be identified postdeformation [21]. This comparison allows us to find the stress necessary to induce twin growth at different spatial positions and, therefore, the strength of the nonlocal interaction term. The value found is consistent with an interaction between residual dislocations at the twin interface and mobile dislocations. This type of model complements density functional theory and MD simulations in which the domain size is limited and typically includes only a single twin formed of less than ten atomic layers [15].

A twin is described by a phase field $\varphi$, varying from 0 (untwinned region) to 1 (twinned region) [22]. Crystal plasticity finite element simulations ((CPFEs) framework $[23,24])$ are performed that include the anisotropy of the elastic properties, plastic deformation due to slip and twin systems and crystal reorientation due to twinning. Existing constitutive equations are able to model only a single twin, whose thickness grows during deformation [25], so are unable to study the emergence of discrete twin patterns typical of many materials.

This paper is organized as follows. We first report the model developed on the basis of the above observations and provide details of the experimental technique. We then compare the characteristics of twins found from experiment and simulations. Finally, we discuss which mechanisms are responsible for twin growth in $\alpha$-uranium.

\section{COMPUTATIONAL METHOD}

The equilibrium equations $\boldsymbol{\nabla} \cdot \boldsymbol{\sigma}=\mathbf{0}$ are solved for the Cauchy stress $\sigma$ using the finite element method [26]. The CPFE framework determines the evolution of the Cauchy stress as a function of time and deformation represented by the displacement vector $\mathbf{u}$ as described in the following.

The Jaumann stress rate, which is the stress rate calculated in the corotational reference frame that follows the crystal rotation, is determined using the tensorial form of Hooke's law [26]:

$$
\stackrel{\nabla}{\sigma}=\mathbb{C} \dot{\varepsilon}_{\mathrm{e}}=\mathbb{C}\left(\dot{\boldsymbol{\varepsilon}}-\dot{\boldsymbol{\varepsilon}}_{\mathrm{p}}\right)
$$
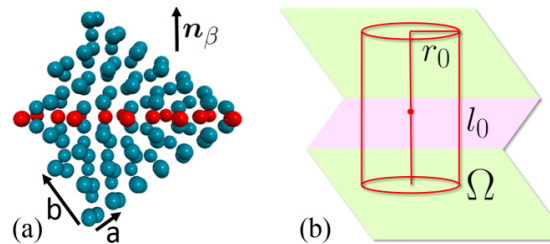

(b)

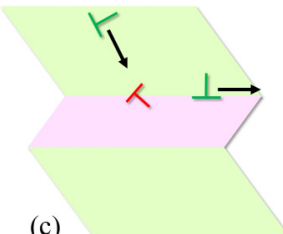

FIG. 1. (a) Twin interface in orthorhombic $\alpha$-uranium, (b) cylindrical integration volume used to calculate the critical RSS (CRSS) for twinning, and (c) dislocation-based mechanism for twin growth.

where $\mathbb{C}$ is the fourth-order elasticity tensor [27] and $\dot{\boldsymbol{\varepsilon}}, \dot{\boldsymbol{\varepsilon}}_{\mathrm{e}}$, and $\dot{\boldsymbol{\varepsilon}}_{\mathrm{p}}$ are the total, elastic, and plastic strain rates, respectively.

The plastic strain rate has contributions from the slip and twin systems [28,29],

$$
\begin{aligned}
\dot{\boldsymbol{\varepsilon}}_{\mathrm{p}}= & \frac{1}{2} \sum_{\alpha=1}^{N_{\text {slip }}} \dot{\gamma}_{\alpha}(\boldsymbol{\sigma})\left(\boldsymbol{s}_{\alpha} \otimes \boldsymbol{n}_{\alpha}+\boldsymbol{n}_{\alpha} \otimes \boldsymbol{s}_{\alpha}\right) \\
& +\frac{1}{2} \dot{\varphi}(\boldsymbol{\sigma}) \gamma_{\beta}^{\mathrm{twin}}\left(\boldsymbol{s}_{\beta} \otimes \boldsymbol{n}_{\beta}+\boldsymbol{n}_{\beta} \otimes \boldsymbol{s}_{\beta}\right) .
\end{aligned}
$$

where $\dot{\gamma}_{\alpha}(\sigma)$ is the dislocation slip rate on slip system $\alpha$, defined by the slip direction $\boldsymbol{s}_{\alpha}$ and slip plane normal $\boldsymbol{n}_{\alpha} \cdot \varphi$ is the twin phase field (defined by the twin direction $\boldsymbol{s}_{\beta}$ and twin plane normal $\boldsymbol{n}_{\beta}$ ) which increases at a rate $\dot{\varphi}$. Whereas the total shear produced by the twin system $\gamma_{\beta}^{\text {twin }}$ is a constant. One twin type [310](130) is considered in the simulations reported here as this is the dominant twinning mode in uranium. If multiple twin systems are active, then this can be readily accounted for by summing the last term in Eq. (2) over $\beta$. The twin interface is shown in Fig. 1(a).

Since $\dot{\boldsymbol{\varepsilon}}_{\mathrm{p}}$ is a nonlinear history-dependent function of the stress, Eq. (1) is solved incrementally using a NewtonRaphson algorithm [26] to find a stress increment consistent with the evolution of the plastic deformation in Eq. (2).

The slip rate $\dot{\gamma}_{\alpha}$ due to dislocation motion is related to the shear stress on the specific slip system, called the resolved shear stress (RSS), using a power-law relationship. Details of the dislocation-based model describing slip as a function of the dislocation density $\rho$ are reported in Ref. [30]. This model, which uses eight slip systems, has been calibrated using neutron diffraction $[30,31]$ and digital image correlation experiments [32].

The novelty here is to incorporate a model for the time evolution of the twin phase field $\varphi$. The twinning process involves the collective displacement of atoms from their initial position in the untwinned lattice to their final position in the twinned lattice. An energy barrier must be overcome after which the system will be driven to the final equilibrium state. This process is described using two terms,

$$
\dot{\varphi}(\sigma, \varphi)=\dot{\varphi}_{S}(\sigma, \varphi)+\dot{\varphi}_{P}(\varphi) .
$$

The first term is stress driven and becomes relevant when the $\operatorname{RSS} \tau_{\beta}$ on the twin system is higher than a CRSS $\tau_{\beta}^{c}(\varphi)$ [33],

$$
\gamma_{\beta}^{\mathrm{twin}} \dot{\varphi}_{S}(\boldsymbol{\sigma}, \varphi)= \begin{cases}\dot{\gamma}_{0}\left|\frac{\tau_{\beta}}{\tau_{\beta}^{c}(\varphi)}\right|^{n}, & \text { if } \tau_{\beta}>0, \\ 0, & \text { if } \tau_{\beta}<0 .\end{cases}
$$


TABLE I. Model parameters.

\begin{tabular}{lcccccc}
\hline \hline$\tau_{0}$ & $\tau_{\text {twin }}^{0}$ & $l_{0}$ & $r_{0}$ & $f$ & $\dot{\gamma}_{0}$ & $n$ \\
\hline $25 \mathrm{MPa}$ & $2000 \mathrm{MPa}$ & $10 \mu \mathrm{m}$ & $10 \mu \mathrm{m}$ & $1 \mathrm{~s}^{-1}$ & $0.001 \mathrm{~s}^{-1}$ & 20 \\
\hline \hline
\end{tabular}

$\tau_{\beta}^{c}(\varphi)$ represents the stress necessary to move the atoms towards the maximum of the energy barrier. $\dot{\gamma}_{0}$ and $n$ are constants. The value of $n=20$ ensures the strain rate independence of the twinning process as observed experimentally [32]. The second term in Eq. (3) represents the force that drives the atoms towards their position in the twinned lattice after the energy barrier is overcome. It is given by

$$
\dot{\varphi}_{P}(\varphi)= \begin{cases}f(1-\varphi), & \text { if } \varphi>\frac{1}{2}, \\ 0, & \text { if } \varphi<\frac{1}{2},\end{cases}
$$

where $f$ is the frequency at which the twinning process completes after the atoms have passed the corresponding energy barrier. It drives the system towards the minimum energy at $\varphi=1$ at which the twin phase field cannot grow further. The value chosen for the characteristic time $1 / f(1 \mathrm{~s})$ is much shorter than the total simulation time but longer than the maximum time increment $\Delta t(0.04 \mathrm{~s})$. This characteristic time is longer than the experimental nucleation time [19] but allows accurate time integration of the twinning nucleation process in the present simulations, in which the strain rate is $10^{-3} \mathrm{~s}^{-1}$. The value of the characteristic time $1 / f$ is also linked to the spatial scale of twin propagation. As shown by the simulations in the following, some twins can propagate through the entire representative volume when the strain is increased from $7 \%$ to $9 \%$ in $20 \mathrm{~s}$, which is a longer time interval than $1 / f$.

The critical RSS contains three contributions:

$$
\tau_{\beta}^{c}(\varphi)=\tau_{0}(\varphi)+\tau_{\text {twin }}(\varphi)+\tau_{\text {dis }},
$$

$\tau_{0}(\varphi)$ accounts for twin nucleation and stress relaxation effect, which leads to strain localization. It is a bilinear function of $\varphi$ and has a minimum corresponding to the maximum of the energy barrier at $\varphi=\frac{1}{2}$ [34],

$$
\tau_{0}(\varphi)= \begin{cases}\frac{\tau_{0}}{4}+\frac{3}{4} \tau_{0}(1-2 \varphi), & \text { if } \varphi<\frac{1}{2}, \\ \frac{\tau_{0}}{4}+\frac{3}{4} \tau_{0}(2 \varphi-1), & \text { if } \varphi>\frac{1}{2} .\end{cases}
$$

A nonlocal term is introduced to describe the effect of a twin on the nucleation stress for a neighboring twin,

$$
\tau_{\text {twin }}(\varphi)=\frac{\tau_{\text {twin }}^{0}}{\Omega}\left(\int_{\Omega} \varphi d V\right),
$$

where $\Omega$ is the volume of a cylindrical region with height $l_{0}$ and radius $r_{0}$, centered on the actual point under consideration as shown in Fig. 1(b). Only integration points where the twin has overcome the energy barrier $\left(\varphi>\frac{1}{2}\right)$ are considered when evaluating the integral. The axis of the cylindrical region is parallel to the twin plane normal. Thus, the integral in Eq. (8) is proportional to the sum of the thickness of the twins in the neighborhood.

The nonlocal term in Eq. (8) is used to model the following process, shown in Fig. 1(c): a mobile dislocation interacts with a twin interface, forming a mobile twinning dislocation and an immobile residual dislocation. The motion of the twinning dislocation forms a new twin layer. Residual dislocations produce a stress field that can prevent further mobile dislocations from reaching the twin interface. The thickness of a twin $t_{\mathrm{twin}}$ is proportional to the number of residual dislocations $N_{\text {res }}$,

$$
t_{\text {twin }}=d_{(130)} N_{\text {res }},
$$

where $d_{(130)}=0.1613 \mathrm{~nm}$ is the (130) planar spacing. For a mobile dislocation far from the twin interface, the stress field of the residual dislocations, which is the linear superposition of their individual stress fields, is proportional to $t_{\text {twin }}$. This justifies the relationship between the additional stress $\tau_{\text {twin }}(\varphi)$ for twin growth and the integral in Eq. (8), which is proportional to the total thickness of neighboring twins. The nonlocal term in Eq. (8) can stop the growth of a twin in a direction parallel to the twin plane normal.

The final term in Eq. (6) $\tau_{\text {dis }}$ represents the interaction between a twin and the mobile dislocations and scales linearly with the total dislocation density $\rho$ as previously determined using digital image correlation experiments [32],

$$
\tau_{\mathrm{dis}}=k_{\beta} \mu_{\beta} b_{\beta} b_{\alpha} \rho,
$$

where $\mu_{\beta}$ is the projected shear modulus and $b_{\beta}$ and $b_{\alpha}$ are the Burgers vectors of the twin and slip systems [30]. The dimensionless constant $k_{\beta}$ in the present simulations is chosen such that $k_{\beta} \mu_{\beta} b_{\beta} b_{\alpha}=0.5 \mathrm{MPa} \mu \mathrm{m}^{2}$. The total dislocation density includes both geometrically necessary dislocations (GNDs) and statistically stored dislocations [35,36].

The three terms in Eq. (6) can be superposed linearly because they represent interactions at different length scales: $\tau_{0}(\varphi)$ is the nucleation stress of a twin embryo with the size of a few atomic layers [15], whereas $\tau_{\text {twin }}(\varphi)$ and $\tau_{\text {dis }}$ represent long-range interaction stresses between dislocations, which can also be superposed linearly [37].

The parameters $\tau_{\text {twin }}^{0}$ and $l_{0}$ describing the twin nucleation stress are determined here by calibrating the model against the experimental results. Parameters used in the following simulations are reported in Table I.

\section{EXPERIMENTAL METHOD}

A flat tensile bar specimen was fabricated by electric discharge machining. Electropolishing was carried out before loading the sample into the scanning electron microscope (SEM). The sample mounted in the tensile machine is shown in Fig. 2. During in situ mechanical testing, the specimen is deformed step by step, allowing for SEM observation and EBSD scanning [38]. EBSD images are taken from a region of size $120 \mu \mathrm{m} \times 120 \mu \mathrm{m}$ inside the red square in Fig. 2 at different stress levels as shown in Fig. 3.

The dynamic formation of discrete twins was observed in the EBSD images at different values of the strain as shown in Fig. 3. The angle that the observed twins form with the horizontal axis and the crystal reorientation allow the active twin system to be identified as the [310](130) type. 


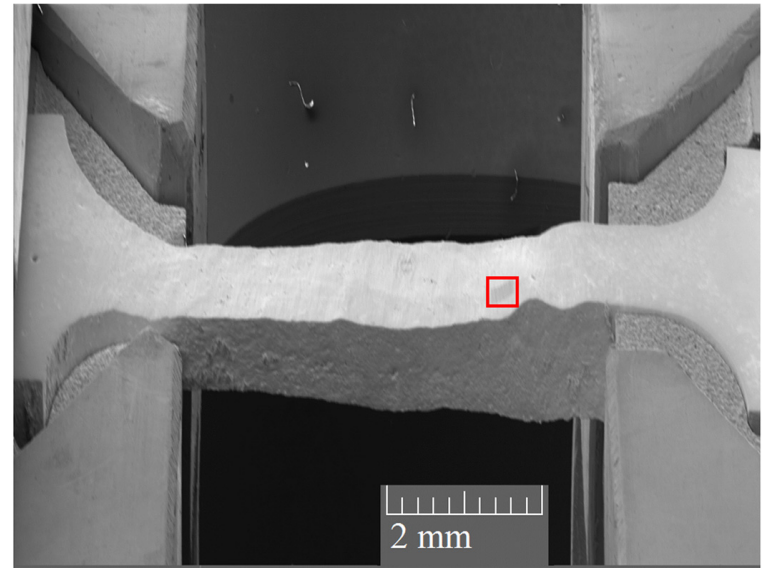

FIG. 2. Sample after deformation and region of interest.

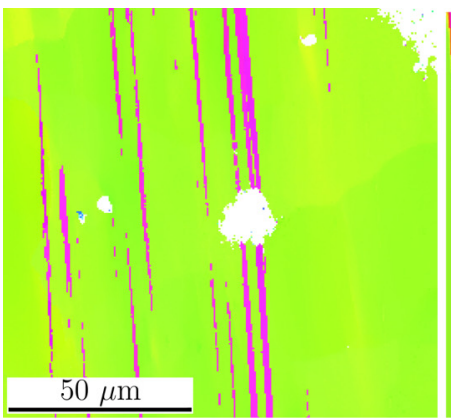

(a)

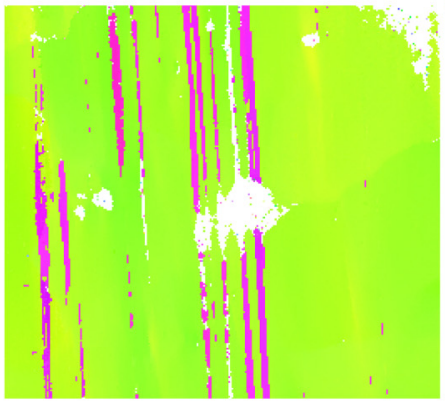

(c)

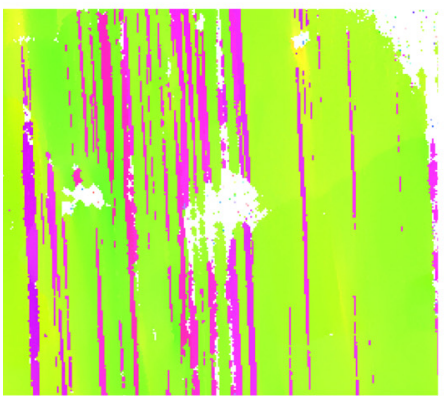

(e)

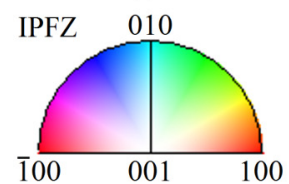

FIG. 3. Comparison between experimental and simulated twins at strain (a) and (b) 7\%, (c) and (d) 9\%, and (e) and (f) $14 \%$.
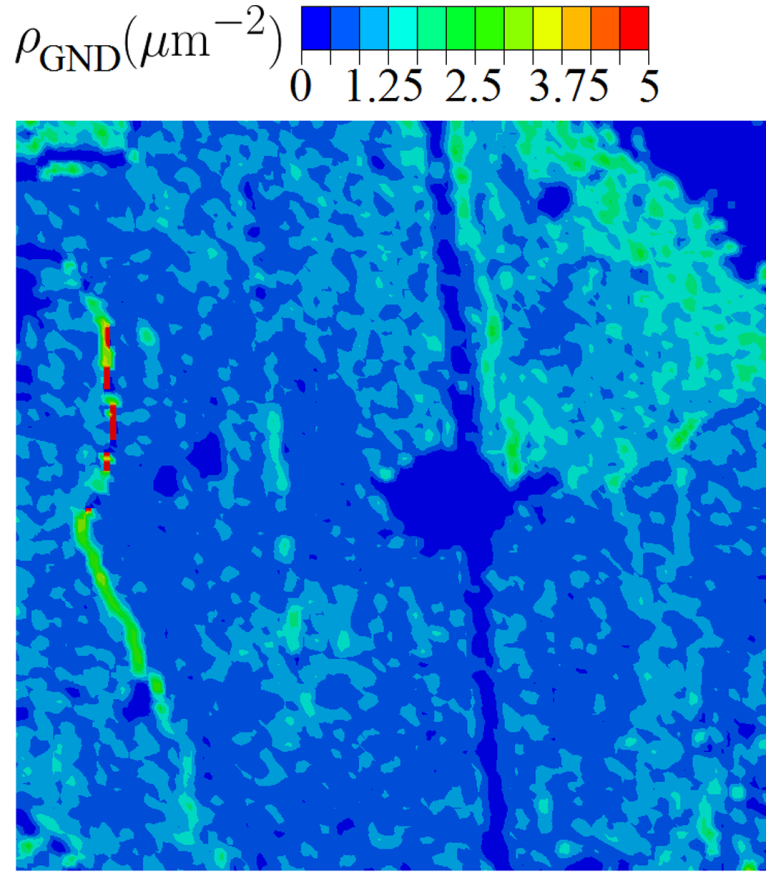

$50 \mu \mathrm{m}$ GND.

FIG. 4. Initial density of geometrically necessary dislocations

\section{RESULTS}

Simulations of the tensile experiment were carried out using a representative volume with the same size as the imaged region in the experiment. The element size is $0.5 \mu \mathrm{m}$. The corresponding time evolution of the twin phase field is shown in Fig. 3.

The EBSD scan before deformation is used to estimate the initial density of geometrically necessary dislocations (GND) in the sample [39] as shown in Fig. 4. This density is added to the dislocation density of the most active slip system in $\alpha$-uranium [100](010).

The initial nucleation of twins in the center of the volume, near the circular nonindexed region, and the prevalence of twins in the left part of the volume at $7 \%$ and $9 \%$ strain are in agreement with the experimental results. This is due to the initial concentration of dislocations in the upper right part of the volume, shown in Fig. 4, which can prevent twin nucleation if the interaction described by Eq. (10) is introduced.

The parameter $l_{0}=10 \mu \mathrm{m}$ can be calibrated by matching the number of twins as a function of the strain as shown in Fig. 5. A larger value of $l_{0}$ leads to a smaller number of twins after deformation because of the longer range of the twin-twin interaction. The uncertainty in the experimental strain is due to the fact that only the displacement applied by the tensile machine is measured, whereas the strain depends on the particular contact conditions between the sample and the clamps. The simulated stress-strain curve is also consistent with the experiment as shown in Fig. 6 and provides a calibration for $\tau_{\text {twin }}^{0}$. One novelty of the present model, compared with existing constitutive equations [33], is the prediction of drops 


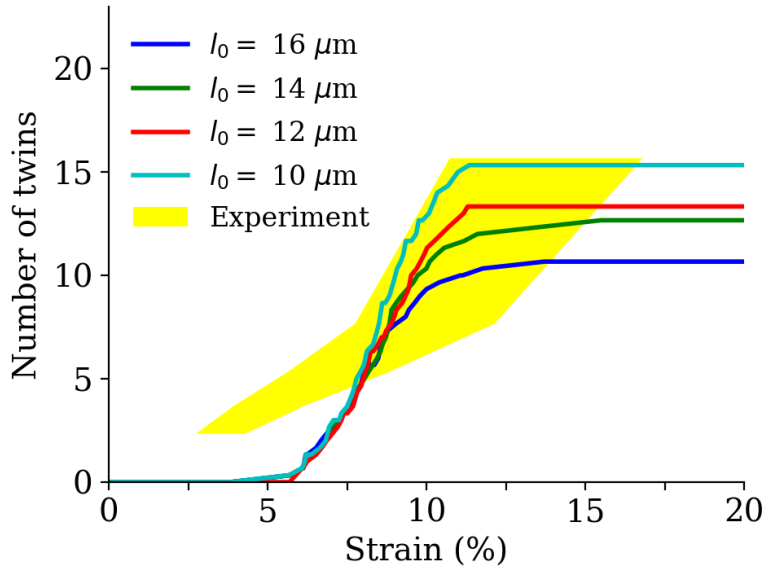

FIG. 5. Number of twins as a function of the strain.

in the local stress, corresponding to the nucleation of new twins. If a value of $\tau_{\mathrm{twin}}^{0}$ is chosen around $250 \mathrm{MPa}$ or lower, no discrete twins form in the simulations but only a single large twin band. On the other hand, if a value greater than $2000 \mathrm{MPa}$ is chosen, the simulated stress-strain curve is no longer consistent with the experiment; specifically, the yield stress and maximum stress are overpredicted. This provides a confidence interval for the parameter $\tau_{\text {twin }}^{0}$.

The mechanism for twin growth that these simulations suggest can be understood by comparing the CRSS for twinning and slip at 7\% strain when new twins are forming in the central part and the left part of the geometry in Fig. 3. From the comparison between the areas in the white dashed square in Figs. 3(b) and 7(a), it can be found that the twin CRSS has maxima between two twin bands and values up to $400 \mathrm{MPa}$, whereas the CRSS for slip is around $80 \mathrm{MPa}$. Indeed, inside the bottom white dashed square, one can observe three twin bands in Fig. 3(b) and two regions where the twinning CRSS has maxima in Fig. 7(a). Therefore, slip is favored in these regions. This is not the case far from preexisting twins where the twinning CRSS is lower and twin nucleation is favored.

Therefore, the proposed mechanism for twin growth is the following: first, twin nucleation occurs far from preexisting GNDs, then, the thickness of the twin embryos grows until

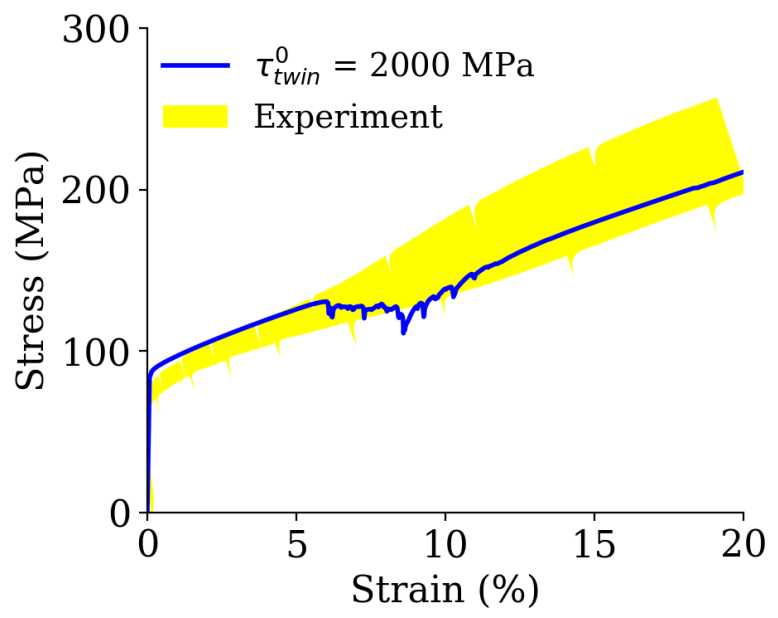

FIG. 6. Stress-strain curve.

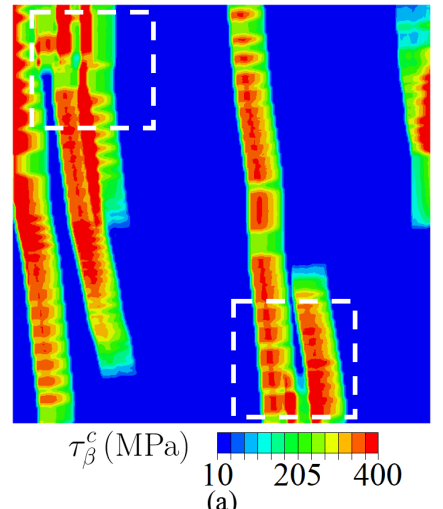

(a)

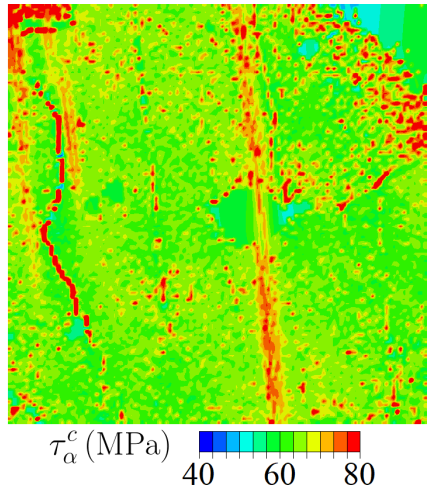

(b)
FIG. 7. CRSS for (a) twin and (b) slip at $7 \%$ strain.

the CRSS for twinning just outside the twinned region exceeds the CRSS for dislocation slip at which point slip becomes the dominant deformation mechanism.

\section{DISCUSSION}

The maximum value of the twinning CRSS (400 MPa) and characteristic length $l_{0}(10 \mu \mathrm{m})$ from these simulations can be used to understand the physical origin of the proposed twin growth mechanism. The characteristic length $l_{0}$ found is typical of a dislocation-based interaction mechanism. Under the hypothesis that the stress field of residual dislocations contributes to the twinning CRSS as described by the term $\tau_{\text {twin }}(\varphi)$ in Eq. (8), its simulated value can be used to estimate the density of residual dislocations per unit length on the twin interface.

The edge dislocations of the most active slip system in $\alpha$-uranium [100](010) can interact with a (130) twin plane as shown in Fig. 8. The stress field given by an array of equally spaced residual dislocations on a (130) twin plane can be calculated by superposing the stress fields of individual dislocations [40].

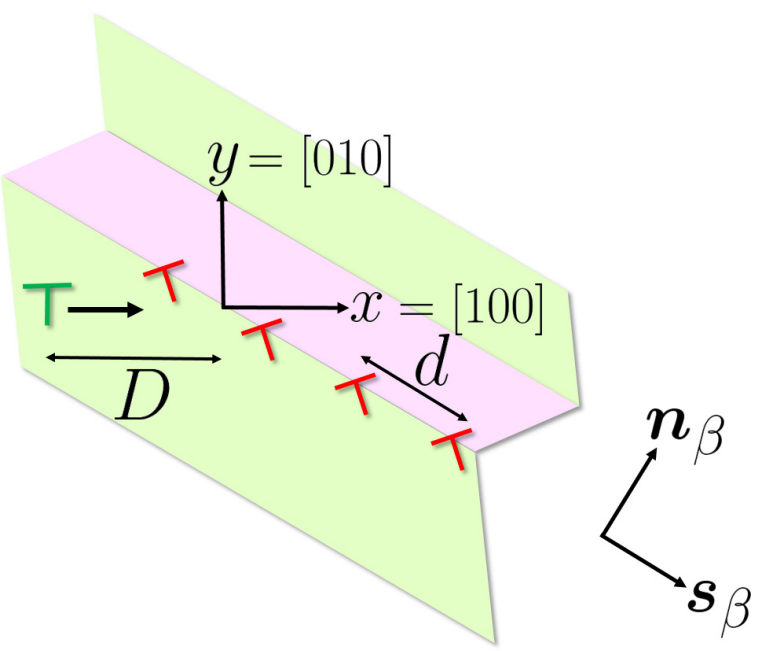

FIG. 8. Array of residual dislocations and mobile dislocation, interacting with a twin interface. 
The Burgers vector of a residual dislocation is given by the difference between the Burgers vectors of the mobile dislocation and of the twinning dislocations. Edge dislocations of the most active slip system in $\alpha$-uranium [100](010) have Burgers vector,

$$
\boldsymbol{b}_{e}=[a, 0,0],
$$

whereas twinning dislocations have a Burgers vector that is on the (130) twin plane,

$$
\boldsymbol{b}_{\mathrm{td}}=\frac{\left(b^{2}-3 a^{2}\right)}{2\left(9 a^{2}+b^{2}\right)}[3 a,-b, 0] .
$$

$a=0.2852$ and $b=0.5865 \mathrm{~nm}$ are the lattice constants of $\alpha$ uranium [33]. Therefore, residual dislocations have Burgers vector $\boldsymbol{b}_{\mathrm{res}}=\boldsymbol{b}_{e}-\boldsymbol{b}_{\mathrm{td}}$,

$$
\boldsymbol{b}_{\mathrm{res}}=\left[a\left(1-\frac{3\left(b^{2}-3 a^{2}\right)}{2\left(9 a^{2}+b^{2}\right)}\right), \frac{b\left(b^{2}-3 a^{2}\right)}{2\left(9 a^{2}+b^{2}\right)}, 0\right] .
$$

Residual dislocations and mobile dislocations have approximately the same Burgers vector (with $\boldsymbol{b}_{\text {res }} \approx \boldsymbol{b}_{e}$ ).

The resolved shear stress induced by a residual dislocation at distance $[\Delta x, \Delta y]$ (with respect to the reference system in Fig. 8) is given by [37]

$$
\sigma_{x y}^{\mathrm{res}}=\frac{\mu\left(\Delta x^{2}-\Delta y^{2}\right)\left(b_{\mathrm{res}}^{x} \Delta x+b_{\mathrm{res}}^{y} \Delta y\right)}{2 \pi(1-v)\left(\Delta x^{2}+\Delta y^{2}\right)^{2}},
$$

where $\mu$ is the shear modulus and $v$ is Poisson's ratio.

Therefore, the repulsive stress field given by the array of residual dislocations as shown in Fig. 8 on a mobile dislocation moving on the (010) slip plane at a distance $D$ from the (130) twin plane can be calculated. Assuming that the mobile dislocation and residual dislocations have coordinates $[-D, 0]$ and $d\left(n-\frac{1}{2}\right)\left[s_{\beta}^{x}, s_{\beta}^{y}\right]$, respectively, where $n$ is a positive integer, $\mathbf{s}_{\beta}$ is the twinning direction, and $d$ is the spacing between residual dislocations, the resolved shear stress is given by

$$
\sigma_{x y}=-\frac{\mu}{2 \pi(1-v) d} \sum_{n=0}^{N_{\mathrm{res}}} \frac{\left\{\left[h+\left(n-\frac{1}{2}\right) s_{\beta}^{x}\right]^{2}-\left(n-\frac{1}{2}\right)^{2}\left(s_{\beta}^{y}\right)^{2}\right\}\left\{b_{\mathrm{res}}^{x}\left[h+\left(n-\frac{1}{2}\right) s_{\beta}^{x}\right]+b_{\mathrm{res}}^{y}\left(n-\frac{1}{2}\right) s_{\beta}^{y}\right\}}{\left\{\left[h+\left(n-\frac{1}{2}\right) s_{\beta}^{x}\right]^{2}+\left(n-\frac{1}{2}\right)^{2}\left(s_{\beta}^{y}\right)^{2}\right\}^{2}}
$$

where $h=D / d$. The sum is taken over $N_{\text {res }}=(120 \mu \mathrm{m} / d)$ dislocations, corresponding to a twin with the size of the observed region. If a larger number of dislocations is used, no large difference in the maximum repulsive stress is found. The repulsive stress field $\left|\sigma_{x y}\right|$ is shown in Fig. 9 as a function of the distance $D$ for different values of the spacing $d$ between residual dislocations.

The maximum stress barrier that the mobile dislocation has to overcome before interacting with the twin plane matches the value $400 \mathrm{MPa}$, found using the continuum model in Fig. 7 (a), if the spacing between residual dislocations is $d=44 \mathrm{~nm}$. When this stress value is reached, twin growth can take place according to the proposed mechanism.

This corresponds the number of residual dislocations per unit length along the twin interface being approximately

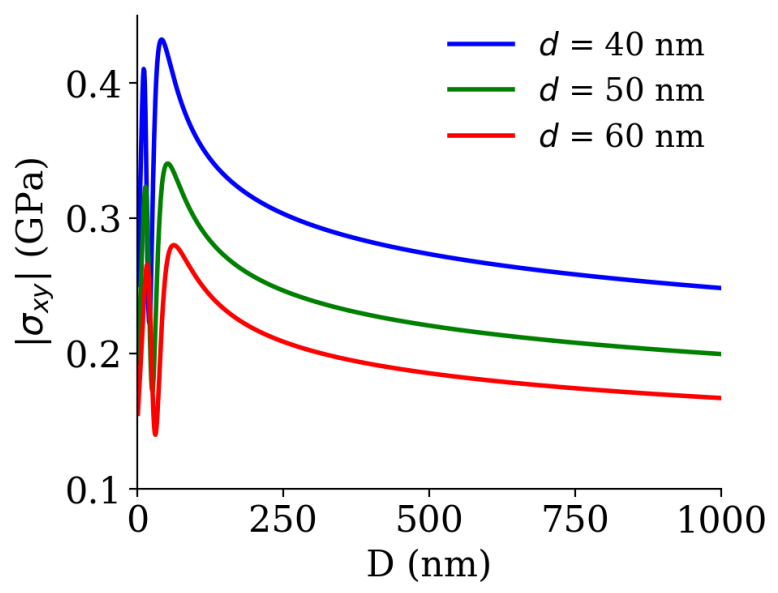

FIG. 9. Repulsive resolved shear stress given by the array of residual dislocations on a mobile dislocation on the (010) slip plane at distance $D$.
$23 \mu \mathrm{m}^{-1}$. According to Eq. (9), since the average twin thickness is approximately $2 \mu \mathrm{m}$, the total number of residual dislocations on a twin interface is about 12400 . Therefore, the total length of a twin is about $12400 / 23 \mu \mathrm{m}^{-1}=539 \mu \mathrm{m}$. This is consistent with the observation in Fig. 3, showing twins crossing the whole observed region $(>120 \mu \mathrm{m})$. It is also consistent with the fact that the total length of a twin in the present sample cannot exceed the sample thickness (760 $\mu \mathrm{m})$. Therefore, the comparison between crystal plasticity simulations and in situ EBSD experiments allows us to find a consistent value for the density of residual dislocations.

The present simulations suggest the additional stress induced by residual dislocations can also prevent the formation of new twin embryos between preexisting twins that are less than $5 \mu \mathrm{m}$ apart. When such a spacing is reached, merging smaller embryos with preexisting twins is not a likely mechanism for twin growth.

Stress-induced nucleation of twinning dislocations at the twin interface is predicted by MD simulations at a stress above $1 \mathrm{GPa}$ [16]. This is not comparable with the twinning CRSS found in the present paper. Therefore, this mechanism for twin growth is also excluded at the present strain rate, which is slower than the one used in MD simulations [16].

\section{CONCLUSIONS}

In conclusion, the model is able to reproduce several discrete twins nucleating during deformation, which is not possible using existing constitutive equations [34]. The correct number of twins as a function of strain and the mechanical properties are consistent with the in situ EBSD experiment.

The main factors that determine twin thickness and spacing are deduced from the comparison between model and experiment: These are the density of residual dislocations at the twin interface and the interaction length between immobile 
residual dislocations and mobile dislocations present between preexisting twins.

This paper shows strong evidence that the additional stress induced by residual dislocations is the mechanism that determines the twin thickness by preventing the further growth of neighboring twins.

This model can help to predict which materials develop thicker twins, but further studies about the nature of residual dislocations on different twin planes will be necessary.

\section{ACKNOWLEDGMENTS}

The authors acknowledge financial support from AWE plc for this research, Program Manager: Dr. J. Askew. E.T. acknowledges support from the Engineering and Physical Sciences Research Council under Fellowship Grant No. EP/N007239/1. P.D.E. and M.N.G. acknowledge support from the Advanced Fuels Campaign of the Fuel Cycle R\&D program in the Office of Nuclear Energy, U.S. Department of Energy.
[1] J. Christian, in The Theory of Transformations in Metals and Alloys, edited by J. Christian (Pergamon, Oxford, 2002), Chap. 20, pp. 859-960.

[2] J. Christian and S. Mahajan, Deformation twinning, Prog. Mater. Sci. 39, 1 (1995).

[3] J. P. Hirth and J. Lothe, Theory of Dislocations, 2nd ed. (Krieger, Malabar, FL, 1982).

[4] S. A. Kibey, L. L. Wang, J. B. Liu, H. T. Johnson, H. Sehitoglu, and D. D. Johnson, Quantitative prediction of twinning stress in fcc alloys: Application to Cu-Al, Phys. Rev. B 79, 214202 (2009).

[5] S. B. Biner and J. R. Morris, The effects of grain size and dislocation source density on the strengthening behavior of polycrystals: a two-dimensional discrete dislocation simulation, Philos. Mag. 83, 3677 (2003).

[6] A. Salem, S. Kalidindi, and S. Semiatin, Strain hardening due to deformation twinning in $\alpha$-titanium: Constitutive relations and crystal-plasticity modeling, Acta Mater. 53, 3495 (2005).

[7] L. Lu, Y. Shen, X. Chen, L. Qian, and K. Lu, Ultrahigh strength and high electrical conductivity in copper, Science 304, 422 (2004).

[8] Q. Li, S. Xue, J. Wang, S. Shao, A. H. Kwong, A. Giwa, Z. Fan, Y. Liu, Z. Qi, J. Ding, H. Wang, J. R. Greer, H. Wang, and X. Zhang, High-strength nanotwinned Al alloys with 9R phase, Adv. Mater. 30, 1704629 (2018).

[9] Y. Guo, H. Abdolvand, T. Britton, and A. Wilkinson, Growth of $\{11 \overline{2} 2\}$ twins in titanium: A combined experimental and modeling investigation of the local state of deformation, Acta Mater. 126, 221 (2017).

[10] A. W. Sleeswyk, Perfect dislocation pole models for twinning in the f.c.c. and b.c.c lattices, Philos. Mag. 29, 407 (1974).

[11] S. Mahajan and G. Chin, Formation of deformation twins in f.c.c. crystals, Acta Metall. 21, 1353 (1973).

[12] A. Ojha, H. Sehitoglu, L. Patriarca, and H. Maier, Twin migration in Fe-based bcc crystals: theory and experiments, Philos. Mag. 94, 1816 (2014).

[13] Y. Liu, P. Tang, M. Gong, R. McCabe, J. Wang, and C. Tomé, Three-dimensional character of the deformation twin in magnesium, Nat. Commun. 10, 3308 (2019).

[14] R. W. Cahn, Twinning and slip in $\alpha$-uranium, Acta Crystallogr. 4, 470 (1951).

[15] S. Ogata, J. Li, and S. Yip, Energy landscape of deformation twinning in bcc and fcc metals, Phys. Rev. B 71, 224102 (2005).

[16] X. Zhao, C. Lu, A. K. Tieu, L. Zhan, L. Pei, and M. Huang, Deformation mechanisms and slip-twin interactions in nanotwinned body-centered cubic iron by molecular dynamics simulations, Comput. Mater. Sci. 147, 34 (2018).
[17] L. Liu, J. Wang, S. K. Gong, and S. X. Mao, High Resolution Transmission Electron Microscope Observation of Zero-Strain Deformation Twinning Mechanisms in Ag, Phys. Rev. Lett. 106, 175504 (2011).

[18] H. Abdolvand and A. J. Wilkinson, Assessment of residual stress fields at deformation twin tips and the surrounding environments, Acta Mater. 105, 219 (2016).

[19] P. G. Oberson and S. Ankem, Why Twins do not Grow at the Speed of Sound All the Time, Phys. Rev. Lett. 95, 165501 (2005).

[20] N. Grilli, K. Janssens, J. Nellessen, S. Sandlöbes, and D. Raabe, Multiple slip dislocation patterning in a dislocationbased crystal plasticity finite element method, Int. J. Plast. 100, 104 (2018).

[21] M. Kumar, I. Beyerlein, R. McCabe, and C. Tomé, Grain neighbor effects on twin transmission in hexagonal close-packed materials, Nat. Commun. 7, 13826 (2016).

[22] C. Liu, P. Shanthraj, M. Diehl, F. Roters, S. Dong, J. Dong, W. Ding, and D. Raabe, An integrated crystal plasticity phase field model for spatially resolved twin nucleation, propagation, and growth in hexagonal materials, Int. J. Plast. 106, 203 (2018).

[23] F. Roters, M. Diehl, P. Shanthraj, P. Eisenlohr, C. Reuber, S. Wong, T. Maiti, A. Ebrahimi, T. Hochrainer, H.-O. Fabritius, S. Nikolov, M. Friák, N. Fujita, N. Grilli, K. Janssens, N. Jia, P. Kok, D. Ma, F. Meier, E. Werner, M. Stricker, D. Weygand, and D. Raabe, DAMASK - the Düsseldorf advanced material simulation kit for modeling multi-physics crystal plasticity, thermal, and damage phenomena from the single crystal up to the component scale, Comput. Mater. Sci. 158, 420 (2018).

[24] R. Baggio, E. Arbib, P. Biscari, S. Conti, L. Truskinovsky, G. Zanzotto, and O. U. Salman, Landau-Type Theory of Planar Crystal Plasticity, Phys. Rev. Lett. 123, 205501 (2019).

[25] M. Ardeljan, I. J. Beyerlein, and M. Knezevic, Effect of dislocation density-twin interactions on twin growth in AZ31 as revealed by explicit crystal plasticity finite element modeling, Int. J. Plast. 99, 81 (2017).

[26] F. Dunne and N. Petrinic, Introduction to Computational Plasticity (Oxford University Press, Oxford, 2006).

[27] E. S. Fisher and H. J. McSkimin, Adiabatic elastic moduli of single crystal alpha uranium, J. Appl. Phys. 29, 1473 (1958).

[28] S. R. Kalidindi, Incorporation of deformation twinning in crystal plasticity models, J. Mech. Phys. Solids 46, 267 (1998).

[29] A. Staroselsky and L. Anand, A constitutive model for hcp materials deforming by slip and twinning: application to magnesium alloy az31b, Int. J. Plast. 19, 1843 (2003). 
[30] N. Grilli, A. C. Cocks, and E. Tarleton, Crystal plasticity finite element modeling of coarse-grained $\alpha$-uranium, Comput. Mater. Sci. 171, 109276 (2020).

[31] N. Grilli, A. Cocks, and E. Tarleton, in Computational Plasticity XV: Fundamentals and Applications, 15th International Conference on Computational PlasticityFundamentals and Applications (COMPLAS), Barcelona, Spain, edited by E. Onate, D. Owen, D. Peric, and M. Chiumenti (International Centre for Numerical Methods in Engineering (CIMNE), Barcelona, Spain, 2019).

[32] N. Grilli, P. Earp, A. C. Cocks, J. Marrow, and E. Tarleton, Characterisation of slip and twin activity using digital image correlation and crystal plasticity finite element simulation: Application to orthorhombic $\alpha$-uranium, J. Mech. Phys. Solids 135, 103800 (2020).

[33] R. McCabe, L. Capolungo, P. Marshall, C. Cady, and C. Tomé, Deformation of wrought uranium: Experiments and modeling, Acta Mater. 58, 5447 (2010).

[34] H. Qiao, M. Barnett, and P. Wu, Modeling of twin formation, propagation and growth in a $\mathrm{Mg}$ single crystal based on crystal plasticity finite element method, Int. J. Plast. 86, 70 (2016).
[35] A. Irastorza-Landa, N. Grilli, and H. V. Swygenhoven, Laue micro-diffraction and crystal plasticity finite element simulations to reveal a vein structure in fatigued cu, J. Mech. Phys. Solids 104, 157 (2017).

[36] A. Irastorza-Landa, N. Grilli, and H. V. Swygenhoven, Effect of pre-existing immobile dislocations on the evolution of geometrically necessary dislocations during fatigue, Modell. Simul. Mater. Sci. Eng. 25, 055010 (2017).

[37] D. Hull and D. Bacon, in Introduction to Dislocations, 5th ed., edited by D. Hull and D. Bacon (Butterworth-Heinemann, Oxford, 2011), Chap. 4 , pp. 63-83.

[38] M. Gussev, P. Edmondson, and K. Leonard, Beam current effect as a potential challenge in SEM-EBSD in situ tensile testing, Mater. Charact. 146, 25 (2018).

[39] S. Das, F. Hofmann, and E. Tarleton, Consistent determination of geometrically necessary dislocation density from simulations and experiments, Int. J. Plast. 109, 18 (2018).

[40] D. Hull and D. Bacon, in Introduction to Dislocations, fourth ed., edited by D. Hull and D. Bacon (Butterworth-Heinemann, Oxford, 2001), Vol. 9, pp. 157-192. 